\title{
Prevalence and antibiotic resistance pattern of extended spectrum beta lactamase producing Escherichia coli isolated from urinary tract infection
}

\author{
Mohsen Rajabnia $^{(\mathbb{1}}$, Mohammad Saad Forghani $^{2}$, Sabah Hasani ${ }^{3}$, Mohammad Bahadoram $^{4}$, Mahsa \\ Mohammadi $^{1}$, Maedeh Barahman ${ }^{5^{*}}$ D
}

'Student Research Committee, Kurdistan University of Medical Sciences, Sanandaj, Iran

${ }^{2}$ Department of Internal Medicine, Faculty of Medicine, Kurdistan University of Medical Sciences, Sanandaj, Iran

${ }^{3}$ Lung Diseases and Allergy Research Center, Kurdistan University of Medical Sciences, Sanandaj, Iran

${ }^{4}$ Medical Student Research Committee \& Social Determinant of Health Research Center, Ahvaz Jundishapur University of Medical

Sciences, Ahvaz, IR Iran

${ }^{5}$ Department of Radiation Oncology, Firoozgar Hospital, Firoozgar Clinical Research Development Center (FCRDC), Iran University of Medical Sciences (IUMS), Tehran, Iran

\section{A R T I C L E I N F O}

Article Type:

Original

Article History:

Received: 17 February 2018

Accepted: 4 July 2018

Published online: 28 July 2018

\section{Keywords:}

Extended-spectrum beta-lactamase Antibiotic resistance

Urinary tract infection

Escherichia coli

Prevalence

\begin{abstract}
A B S T RA C T
Introduction: Urinary tract infection (UTI) due to extended spectrum beta-lactamase (ESBL)-producing bacteria including Escherichia coli has become widespread. Studies have shown a trend toward higher mortality, longer hospitalization, greater hospital expenses and reduced rates of clinical and microbiologic response in ESBL UTI.

Objectives: The aim of this study is to determinate the prevalence and antibiotic resistance pattern of ESBL producing E. coli isolated from UTI.

Patients and Methods: This cross-sectional study was conducted on 3126 samples. Urine specimens were cultured on Eosin Methylene Blue (EBM) and blood agar. The disk diffusion standard method (Kirby Bauer) was used to test the susceptibility of the drug on MullerHinton agar plates and results were reviewed based on Clinical and Laboratory Standards Institute (CLSI) criteria. The reviewing of ESBL-producing uropathogens was carried out using Combined Disk Test (CDT) by using cefotaxime (CTX; $30 \mu \mathrm{g}$ ) and cefotaximeclavulanic acid (CTX; $30 \mu \mathrm{g} / \mathrm{CA}: 10 \mu \mathrm{g}$ ) disks and CLSI protocol.

Results: Out of $291 \mathrm{E}$. coli isolates, 108 (37.11\%) are ESBL-producer and 183 (62.89\%) are non-ESBL-producer. Among ESBL-producing E. coli, the highest antibiotic resistance was observed with cefotaxime (100\%), amoxicillin (97.22\%) and piperacillin (96.3\%) and the highest antibiotic sensitivity was observed with meropenem (93.5\%), nitrofurantoin (81.48\%) and gentamicin (55.56\%).

Conclusion: We recommended that cephalosporins, penicillins and cotrimoxazole are not suggested in the treatment of ESBL-producing E. coli. On the other hand, carbapenems as a first line and aminoglycosides as the next step in the treatment of ESBL-producing E. coli are recommended.
\end{abstract}

Implication for health policy/practice/research/medical education:

Knowing the prevalence and antibiotic resistance pattern of extended spectrum beta lactamase producing Escherichia coli as the most common urinary tract infection pathogen, in addition to alert to health system policymakers about increasing antibiotics resistance, helps therapists to prescribe appropriate antibiotics to treat them.

Please cite this paper as: Rajabnia M, Forghani MS, Hasani S, Bahadoram M, Mohammadi, Barahman M. Prevalence and antibiotic resistance pattern of extended spectrum beta lactamase producing Escherichia coli isolated from urinary tract infection. J Renal Inj Prev. 2019;8(2):78-81. DOI: 10.15171/jrip.2019.15.

\section{Introduction}

Urinary tract infection (UTI) is one of the most common infectious disease that affects about more than 150 million people every year (1). Escherichia coli is the most common urinary tract pathogen, accounting for $50 \%$ to $90 \%$ of UTI isolates (2). E. coli as an enterobacteriaceae 
member is gram negative and facultative anaerobic bacterium (3). $\beta$-lactam antibiotics (beta-lactam antibiotics) are the commonly prescribed antibiotics for UTI. Beta-lactam antibiotics are a class of broad-spectrum antibiotics, consisting of all antibiotic agents that contain a beta-lactam ring in their molecular structure (4). Betalactamases are enzymes that hydrolyze beta-lactam ring, inactivating the antibiotic (5). Extended-spectrum betalactamase (ESBL) are enzymes that confer resistance to most common beta-lactam antibiotics such as penicillins, cephalosporins and monobactam (6). ESBL-producing bacteria were first reported in 1983, and now infection due to ESBL-producing bacteria including $E$. coli has become widespread (7). Studies have shown a trend toward higher mortality, longer hospitalization, greater hospital expenses and reduced rates of clinical and microbiologic response in ESBL UTI $(8,9)$.

\section{Objectives}

The aim of this study is to determinate the prevalence and antibiotic resistance pattern of ESBL-producing E. coli isolated from UTI.

\section{Patients and Methods \\ Patients}

This cross-sectional study was conducted on all urine samples (3126 samples) sent to the cultivating to the central laboratory of Tohid hospital in Sanandaj from October 2016 to September 2017.

\section{Intervention and biochemical measurements}

Urine specimens were collected from the midstream urine samples in sterile containers and by using a calibrated loop $(0.01 \mathrm{~mL})$, they were cultured in sterile conditions on Eosin Methylene Blue (EBM). The Blood agar and then samples were examined at $37^{\circ} \mathrm{C}$ after $18-24$ hours incubation. The samples that number of colonies grown on their culture medium were more than $10^{5} \mathrm{CFU} / \mathrm{mL}$ were examined as a positive urine culture to identify genus and bacterial species based on standard methods. After the final diagnosis, the disk diffusion standard method (Kirby Bauer) was used to test the susceptibility of the drug on Muller-Hinton agar plates. Creating or not creating and the size of inhibition zone around the disk were reviewed based on the Clinical and Laboratory Standards Institute (CLSI) criteria. The most commonly used antibiotics in UTIs were reviewed in studied antibiogram. The reviewing of ESBL-producing uropathogens was carried out using Combined Disk Test (CDT) and CLSI protocol. At first, a microbial suspension equivalent to half McFarland was prepared from pure bacterial culture and it was cultured on Muller-Hinton agar medium by sterile swab. Then, the cefotaxime (CTX; $30 \mu \mathrm{g}$ ) and cefotaxime-clavulanic acid (CTX; $30 \mu \mathrm{g} / \mathrm{CA} ; 10 \mu \mathrm{g}$ ) disks were placed at the distance of at least $2.5 \mathrm{~cm}$ from each other in medium. After 24 hours of incubation at $37^{\circ} \mathrm{C}$, producing ESBL was studied. If the inhibition zone around the cefotaxime-clavulanic acid was equal $3 \mathrm{~mm}$ or it was greater than inhibition zone around cefotaxime, that bacteria was considered as ESBL producing.

\section{Ethical issues}

The study was in accordance with the Declaration of Helsinki and all participants gave their informed consent to enter the study. The study was approved by the Research Committee and the Ethical Committee of the Kurdistan University of Medical Sciences. (Ethic code: IR.MUK. REC.1397.5014).

\section{Data analysis}

The data were analyzed by SPSS 23 software. To compare qualitative variables, chi-square test was applied. Level of significance was $P<0.05$.

\section{Results}

Of 3126 studied urine samples, the results of 708 cultures (22.64\%) were positive. Among uropathogens, E. coli was the most common bacteria (41.10\%). On the other hand, 163 bacteria (23.02\%) produced ESBL (Figure 1). Out of 291 E. coli isolates, $108(37.11 \%)$ are ESBL-producer and

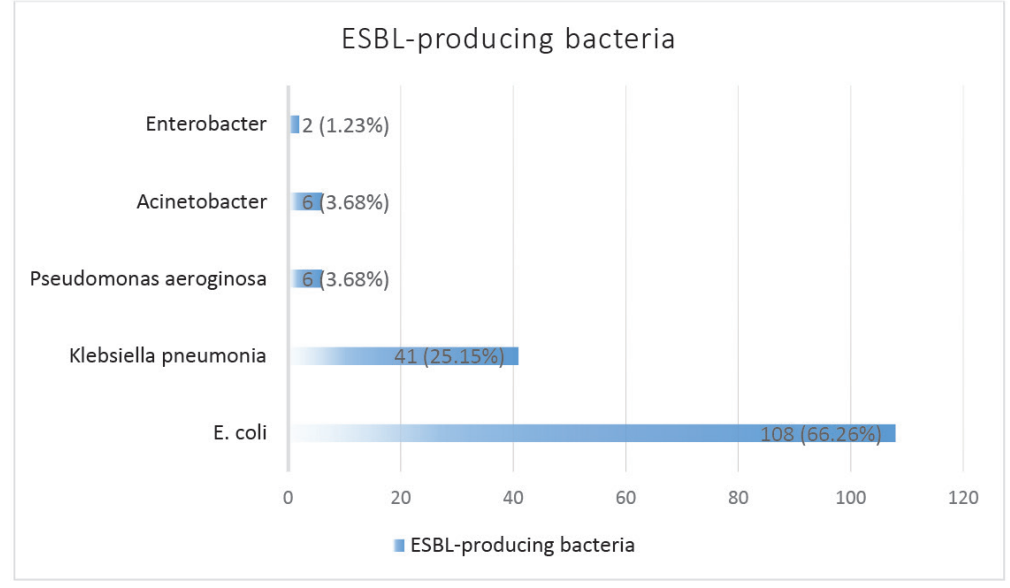

Figure 1. The frequency of ESBL-producing bacteria. 


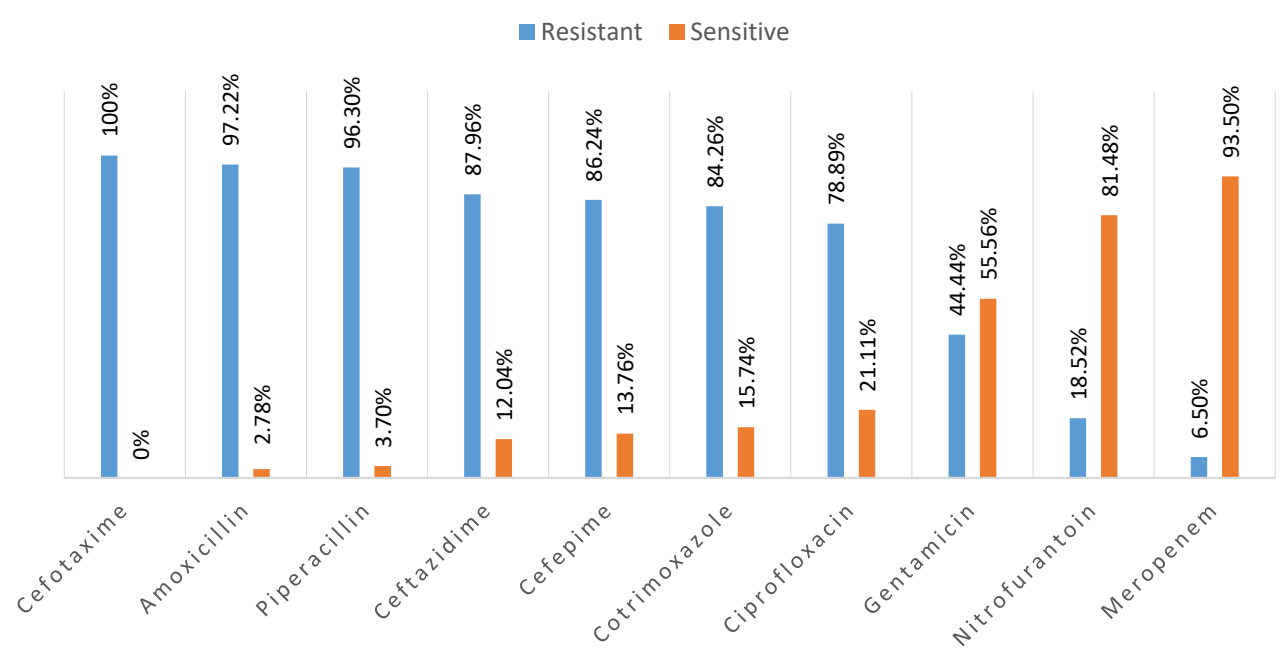

Figure 2. Antimicrobial resistance pattern of ESBL-producing E. coli.

183 (62.89\%) are non-ESBL-producer.

According to the result of antibiogram, the highest antibiotic resistance among ESBL-producing $E$. coli was observed with cefotaxime (100\%), amoxicillin (97.22\%) and piperacillin (96.3\%). The highest antibiotic sensitivity among ESBL-producing E. coli was observed with meropenem (93.5\%), nitrofurantoin (81.48\%) and gentamicin (55.56\%) (Figure 2).

\section{Discussion}

This study showed that $163(23.02 \%)$ bacteria isolated from urinary culture were ESBL-producing. Out of 291, E. coli isolates, 108 (37.11\%) are ESBL-producer and $183(62.89 \%)$ are non-ESBL-producer. Other studies reported a prevalence of ESBL-producer $E$. coli isolates $46.87 \%$ (10), $82.6 \%$ (11) and $21.4 \%$ (12) respectively. This different results may be due to the difference in the risk factors include recent antibiotic therapy, administration of corticosteroids, hospitalization (13).

According to the result of antibiogram, the highest antibiotic resistance among ESBL-producing E. coli was observed with cefotaxime (100\%), amoxicillin (97.22\%) and piperacillin (96.3\%). The highest antibiotic sensitivity was observed with meropenem (93.5\%), nitrofurantoin $(81.48 \%)$ and gentamicin (55.56\%). This study showed that ESBL-producing E. coli were resistant to penicillins and cephalosporins and sensitive to carbapenem, nitrofurantoin and aminoglycoside. Our findings are similar to other studies (14-16) (Table 1).

\section{Conclusion}

Given the dramatic increase in the prevalence of ESBLproducing E. coli and its consequences, including higher mortality, longer hospitalization, greater hospital expenses and reduced rates of clinical and microbiologic response, evaluation of their prevalence and antibiotic resistance pattern are recommended. According to the results of our study, it is recommended that cephalosporins, penicillins and cotrimoxazole are not administered in the treatment of ESBL-producing E. coli. On the other hand, carbapenems as a first line and aminoglycosides as the next step in the treatment of ESBL-producing E. coli are recommended.

Limitations of the study

The limitation of this study was that the standard and correct method is the MIC method, while this method has

Table 1. Comparison of results of studies

\begin{tabular}{|c|c|c|c|}
\hline This study & Yadav et al (14) & Gngane et al (15) & Fernando et al (16) \\
\hline \multicolumn{4}{|l|}{ Antibiotic resistance } \\
\hline Cefotaxime (100\%) & Cephalexin (78.86\%) & Ampicillin (100\%) & Ceftriaxone (100\%) \\
\hline Amoxicillin (97.22\%) & Nalidixic acid (73.98\%) & Cotrimoxazole (93.80\%) & Ceftazidime (100\%) \\
\hline Piperacillin (96.30\%) & Cotrimoxazole (71.54\%) & Nalidixic acid (89.70\%) & Ciprofloxacin (90.10\%) \\
\hline \multicolumn{4}{|l|}{ Antibiotic sensitive } \\
\hline Meropenem (93.50\%) & Imipenem (62.60\%) & Imipenem (100\%) & Meropenem (96.10\%) \\
\hline Nitrofurantoin (81.48\%) & Amikacin (60.97\%) & Piperacillin-Tazobactam (88.10\%) & Imipenem (73.70\%) \\
\hline Gentamicin (55.56\%) & Nitrofurantoin (43.90\%) & Nitrofurantoin (71.30\%) & Nitrofurantoin (45.90\%) \\
\hline
\end{tabular}


not been used in this study.

\section{Acknowledgments}

The authors would like to thank Research Consulting center (RCC) for their participation in manuscript revision.

\section{Authors' contribution}

All authors passed four criteria for authorship contribution based on recommendations of the International Committee of Medical Journal Editors. MRC and MSF conducted the research. MM and AF wrote the primary draft. $\mathrm{MOB}$ And $\mathrm{MB}$ prepared the final paper. $\mathrm{SH}$ conducted the final check of the paper. All authors read and signed the final paper.

\section{Conflicts of interest}

The authors declare that they do not have any conflict of interest.

\section{Ethical considerations}

Ethical issues (including plagiarism, data fabrication, double publication) have been completely observed by the authors.

\section{Funding/Support}

This research was funded by Kurdistan University of Medical Sciences.

\section{References}

1. Almomani BA, Hayajneh WA, Ayoub AM, Ababneh MA, Al Momani MA. Clinical patterns, epidemiology and risk factors of community-acquired urinary tract infection caused by extended-spectrum beta-lactamase producers: a prospective hospital case-control study. Infection. 2018 May 10. doi: 10.1007/s15010-018-1148-y.

2. Hadifar S, Moghoofei M, Nematollahi S, Ramazanzadeh R, Sedighi M, Salehi-Abargouei A, et al. Epidemiology of multidrug resistant uropathogenic Escherichia coli in Iran: a Systematic Review and Meta-Analysis. Jpn J Infect Dis. 2017;70:19-25. doi: 10.7883/yoken.JJID.2015.652.

3. Van Duin D. Carbapenem-resistant Enterobacteriaceae: What we know and what we need to know. Virulence. 2017;8:379-382. doi: 10.1080/21505594.2017.1306621.

4. Ruben F, Paula A, Cristina P. Beta-Lactams: chemical structure, mode of action and mechanisms of resistance. Rev Med Microbiol. 2013;24:7-17. doi: 10.1097/ MRM.0b013e3283587727.

5. Pérez Heras I, Sanchez-Gomez JC, Beneyto-Martin P, Ruano-de-Pablo L, Losada-Pinedo B. Community-onset extended-spectrum $\beta$-lactamase producing Escherichia coli in urinary tract infections in children from 2015 to 2016: Prevalence, risk factors, and resistances. Medicine (Baltimore). 2017;96:e8571. doi: 10.1097/ MD.0000000000008571.

6. Picozzi SC, Casellato S, Rossini M, Paola G, Tejada M, Costa E, et al. Extended-spectrum beta-lactamase-positive Escherichia coli causing complicated upper urinary tract infection: Urologist should act in time. Urol Ann. 2014;6:107-12. doi: 10.4103/0974-7796.130536.

7. Kim YH, Yang EM, Kim CJ. Urinary tract infection caused by community-acquired extended-spectrum $\beta$-lactamaseproducing bacteria in infants. J Pediatr (Rio J). 2017;93:260266. doi: 10.1016/j.jped.2016.06.009.

8. Ramphal R, Ambrose PG. Extended-spectrum betalactamases and clinical outcomes: current data. Clin Infect Dis. 2006;42 Suppl 4:S164-72. doi: 10.1086/500663.

9. Savatmorigkorngul S, Poowarattanawiwit P, Sawanyawisuth K, Sittichanbuncha Y. Factors associated with extended spectrum B-lactamase producing Escherichia coli in community-acquired urinary tract infection at hospital emergency department, Bangkok, Thailand. Southeast Asian J Trop Med Public Health. 2016;47:227-33.

10. Kulkarni DM, Bardapurkar SA, Nilekar SL, More SR. Prevalence of extended-spectrum beta-lactamaseproducing E. coli and Klebsiella species in urinary isolates. IOSR-JDMS. 2016;15:26-29. doi: 10.9790/0853-1506052629

11. Singh N, Pattnaik D, Neogi DK, Jena J, Mallick B. Prevalence of ESBL in Escherichia coli isolates among ICU patients in a tertiary care hospital. J Clin Diagn Res. 2016;10:DC19DC22. doi: 10.7860/JCDR/2016/21260.8544

12. Datta P, Gupta V, Sidhu S, Chander J. Community urinary tract infection due to ESBL producing E. coli: epidemiology and susceptibility to oral antimicrobials including Mecillinam. Nepal Journal of Medical Sciences. 2014;3:5-7. doi: 10.3126/njms.v3i1.10341

13. Lee JA, Kang CI, Joo EJ, Ha YE, Kang SJ, Park SY, et al. Epidemiology and clinical features of community-onset bacteremia caused by extended-spectrum $\beta$-lactamaseproducing Klebsiella pneumoniae. Microb Drug Resist. 2011;17:267-73. doi: $10.1089 / \mathrm{mdr} .2010 .0134$.

14. Yadav Kh, Prakash S. Screening of ESBL producing multidrug resistant E. coli from urinary tract infection suspected cases in southern Terai of Nepal. J Infect Dis Diagn. 2017;2:116.

15. Gangane R, Firdous J. Isolation and antibiotic sensitivity pattern of extended spectrum beta lactamase (ESBL) producing Escherichia coli isolated from urinary tract infection. Int J Curr Microbiol Appl Sci. 2017;6:279-286. doi: $\quad 10.20546 /$ ijcmas.2017.606.034.

16. Fernando MM, Luke WA, Miththinda JK, Wickramasinghe RD, Sebastiampillai BS, Gunathilake MP, et al. Extended spectrum beta lactamase producing organisms causing urinary tract infections in Sri Lanka and their antibiotic susceptibility pattern -A hospital based cross sectional study. BMC Infect Dis. 2017;17(1):138. doi: 10.1186/ s12879-017-2250-y.

Copyright $\odot 2019$ The Author(s); Published by Nickan Research Institute. This is an open-access article distributed under the terms of the Creative Commons Attribution License (http://creativecommons.org/licenses/by/4.0), which permits unrestricted use, distribution, and reproduction in any medium, provided the original work is properly cited. 